\title{
The effect of aerobic exercises on lipid profile of patients with human immunodeficiency virus infection undergoing highly active antiretroviral therapy: a protocol for a systematic review
}

\author{
Ebuka Miracle Anieto,*, Chidimma Amarachukwu Nwankwo', Samuel Ugochukwu Jumbo², Michael Ebe Kalu \\ 'Department of Medical Rehabilitation, Faculty of Health Sciences and Technology, University of Nigeria, Enugu Campus, Enugu, Nigeria \\ ${ }^{2}$ Faculty of Health and Rehabilitation Sciences, Elborn College, Western University, London, ON, Canada \\ ${ }^{3}$ School of Rehabilitation, McMaster University, Hamilton, ON, Canada
}

Patients living with human immunodeficiency virus (HIV) infection who are treated with highly active antiretroviral therapy (HAART) often experience metabolic changes that have an adverse effect on their overall health status. This review will assess the effect of aerobic exercises on the lipid profile of individuals with HIV infection undergoing HAART. Cochrane Library, MEDLINE, CINAHL (Cumulative index to Nursing and Allied Health Literature), OVID, ProQuest, AMED (Allied and Complementary Medicine Database), PsycINFO, Web of Science Core Collection and Pedro databases will be searched until June 2018. Studies that investigated the effects of aerobic exercises on the lipid profile of HAART treated patients with HIV will be included. Two reviewers will screen all articles for eligibility and independently evaluate the risk of bias, complete quality assessment and extract data for all included articles. Homogenous quantitative outcome data will be analyzed using a random effect model of meta-analysis with results presented as relative risk for dichotomous variable and standardized mean for continuous variable. Heterogeneous qualitative data will be analyzed using narrative synthesis. This systematic review will provide evidence about the effectiveness of aerobic exercises in managing HAART related dyslipidemia in patients with HIV infection and thus provide the impetus for more structured exercise interventions.

Keywords: Aerobic exercises, Dyslipidemia, Highly active retroviral therapy, Human immunodeficiency virus infection, Lipids, Systematic review

\section{INTRODUCTION}

The advancement in the use of highly active antiretroviral therapy (HAART) has significantly reduced morbidity and mortality in the population of people living with human immunodeficiency virus (HIV) infection (Palella et al., 1998). However, HAART which is the current standard treatment for HIV have been associated with several deleterious side effects of which lipid profile disorders is one (Carr et al., 1998; Schambelan et al., 2002; Souza et al., 2013). Symptoms of these side effects usually includes hyperlipedemia (i.e., increased triglycerides, low density lipoprotein), decreased high-density lipoprotein cholesterol (HDL-C) among

others (Anuurad et al., 2009; Thompson et al., 2011). Lipid abnormalities such as low HDL-C is a widely recognized independent predictor of cardiovascular diseases in patients living with HIV (Kelesidis et al., 2013).

Aerobic exercise trainings have been reported to have mitigating effect on the metabolic changes that emanate from treatment with HAART (Katzmarzyk et al., 2003). Evidence has shown that physical activities that expend 1,200 to 2,200 kcal/wk are often effective at increasing HDL-C levels from 2 to $8 \mathrm{mg} / \mathrm{dL}$, decreasing triglyceride (TG) levels by 5 to $38 \mathrm{mg} / \mathrm{dL}$, decreasing total cholesterol and low-density lipoprotein (LDL) (Ahmed et al., 2012). To date, evidence on the effectiveness of aerobic exercise

${ }^{*}$ Corresponding author: Ebuka Miracle Anieto (iD https://orcid.org/0000-0002-8160-9701 Department of Medical Rehabilitation, Faculty of Health Sciences and Technology, University of Nigeria, Enugu Campus, 40006 Enugu, Nigeria Tel: +2348069535182, E-mail: ebuka.anieto.183360@unn.edu.ng

Received: May 14, 2018 / Accepted: July 14, 2018

This is an Open Access article distributed under the terms of the Creative Commons Attribution Non-Commercial License (http://creativecommons.org/licenses/by-nc/4.0/) which permits unrestricted non-commercial use, distribution, and reproduction in any medium, provided the original work is properly cited. 
training as a single exercise regime in management of HAART-related dyslipedemia is lacking. As a result, there are disagreements pertaining interventions centered on using aerobic exercises as a management strategy. Therefore, systematically evaluating evidence on the effectiveness of aerobic exercise in mitigating the effect of HAART on the lipid profile of patients with HIV infection is warranted.

This systematic review is therefore aimed at determining the effectiveness of aerobic exercises in the management of HAARTinduced dyslipedemia in patients living with HIV. Specifically, the objectives are: (a) to determine the effect of aerobic exercise in mitigating the side effects of HAART on serum lipids of patients with HIV infection, and (b) to determine the effect of aerobic exercises in improving the overall quality of life of HAART-treated patients with HIV infection.

\section{MATERIALS AND METHODS}

\section{Design}

The systematic review and subsequent network of meta-analysis will be conducted following the method outline in the Preferred Reporting items for systematic reviews and Meta-Analyses Protocol (PRISMA-P) 2015 guidelines (Moher et al., 2015; Shamseer et al., 2015). Studies meeting the broad eligibility criteria will be included and described. The studies that meet the narrower criteria will finally be reviewed and described.

\section{Eligibility criteria}

The following eligibility criteria will be applied in selecting studies.

\section{Inclusion criteria}

1. Language: Only papers published in English language will be included.

2. Subjects: Adults ( $\geq 18$ years old) with HIV infection receiving HAART will be included.

3. Interventions: Aerobic exercise intervention, defined as physical activity of low to high intensity requiring free oxygen and utilization of oxygen to sufficiently meet energy demands during exercise through aerobic metabolism (Pang et al., 2006). No particular restriction will be considered as regarding a particular form of aerobic exercise and its FITT parameter (frequency, intensity, time, and type). Multifactorial interventions that combines aerobic exercise with other interventions such as medications, nutrition, health education will be included provided the effect of the aerobic exercise can be determined.

4. Study setting: No particular restriction will be drawn regarding settings of studies to be included. Therefore, studies conducted in clinics, health centers, rehabilitation homes, hospitals or community settings will be included.

5. Study designs: Randomized clinical trials (RCTs) and nonRCTs including quasi-RCTs, controlled clinical trials (crossover trials), before and after trials (controlled and noncontrolled) that investigated the effects of these interventions will be included.

6. Comparators/control: Any type of control will be included. Intervention studies devoid of control groups will equally be included.

7. Outcomes: Primary outcome measure will be blood/serum lipid profile. Secondary outcome will include quality of life measured with generic validated tools. Definitions of outcomes will be extracted as reported in the included studies.

\section{Exclusion criteria}

Article will be excluded if;

1. The interventions did not include any form of aerobic exercise.

2. It is an opinion paper, case reports, any reviews (systematic, scoping or narrative).

In a case where authors published several articles using the same data set, only the article with a more comprehensive data will be included in this review.

\section{Search strategy}

The strategy for identifying relevant literature has been developed and piloted in consultation with a health-research librarian (Appendix 1). The search strategy was developed based on the instructions in Cochrane handbook for systematic reviews of interventions (Higgins and Green, 2011) and the center for review and dissemination recommendation for health care review (Akers et al., 2009). A search of bibliographic databases and will be carried out with the search strategy; searches will involve a combination of terms of medical subject headings (MeSh) and keywords in the title, abstract and text for the population, intervention, comparison and outcomes.

Multiple database including Cochrane Library, MEDLINE, CINAHL (Cumulative index to Nursing and Allied Health Literature), OVID, ProQuest health and medical complete, AMED (Allied and Complementary Medicine Database), PsycINFO, Web of Science Core Collection and PEDRO (Physiotherapy Evidence da- 
tabase) will be searched to identify the appropriate articles. In addition, hand searching of the reference list of the identified studies, conference proceeding, directory of open-access repository websites and trial register (e.g., http://www.clinicaltrial.gov, http://www.opendor.org) will also be searched.

\section{Study selection}

Two independent review authors will do the study selection on the basis of the study eligibility criteria. First, all results will be collected, and duplicates removed using Refworks, subsequently Covidence (Covidence, Melbourne, VIC, Australia) will be utilized to manage articles throughout the screening and study selection process. Second, all titles and abstracts will be independently screened by two review authors and possible relevant articles will be then reviewed independently in full-text. A third review author will resolve any possible inclusion disagreements. The level of agreement on study eligibility will be tested using the kappa statistics and 95\% confidence interval (CI). Furthermore, authors of studies will be reached through a round of three email attempts, to elucidate any problems that relates to including a study especially when a decision could not be made based on available information. In the case of no response from the author, the study will be excluded and the reason for exclusion reported. A flow chart (PRISMA diagram) will be utilized to trace the overall process.

\section{Quality appraisal for included studies}

The risk of bias in the selected studies will be assessed by two author reviewers using the Cochrane Collaboration risk of bias tool (Higgins and Green, 2011). Each of the included studies will be assessed and graded as 'high risk' or 'low risk' as recommended by Moher et al. (2015). Studies will be subsequently rated as trials with low quality (i.e., having high risk of bias) or trials with high quality (i.e., having low to moderate risk of bias) if there were three or more, or fewer than three recognizable sources of bias respectively.

\section{Data collection processes}

The Cochrane Consumers and Communication Review Group Data Extraction Template will be utilized for the purpose of data extraction from included studies (Cochrane Consumers and Communication Review Group, 2007). Two reviewers will carry out the extraction of data independent of each other. Any disagreement during data extraction will be tackled in research meetings including the last review author in attendance.

\section{Data items}

The following variables will be collected from the included articles: (a) the article meta data including authors name, principal author's country, study setting, study purpose; (b) participants' characteristics such as age, gender composition; (c) study methodology including sample size, sampling method, number of participants enrolled, number of participants included in the analysis; (d) interventions including type of aerobic exercise, exercise parameters-FITT, length of intervention and follow up, characteristics of the exercise coach primary and secondary outcome measures and tools; (e) information on HAART intake and; (f) Data analysis method, findings, recommendation and conclusions.

\section{Outcomes and prioritisation}

The primary outcome will include serum concentration of lipids (HDL-C, LDL-C, total cholesterol, and TGs). This is chosen as the primary outcome as serum lipid profile may be relevant across conditions that are HAART-induced, potentially enabling a meta-analysis. Also, this review aims at identifying the effect of aerobic exercise intervention on serum lipids, thus reducing the risk of cardiovascular events in HAART-treated patients with HIV infection. Secondary outcome will include quality of life. The secondary outcome was selected because it plays a major role in the overall health status of patients with chronic conditions. For crossover trials, data will be extracted from the first period only to avoid a cross-over effect.

\section{Risk of bias assessment in individual studies}

The risk of bias for each of the intervention studies will be assessed using The Cochrane Collaboration Tool for Risk of Bias Assessment (Higgins and Green, 2011). A calibration meeting between two author reviewers will be done to ascertain the risk of bias of the included studies. Each of the review authors will independently examine the risk of bias in all the included articles with the Cochrane Collaboration Tool for Risk of Bias Assessment in these six key domains: (a) selection bias (random sequence generation and allocation concealment); (b) performance bias (blinding of personnel and participants); (c) detection bias (blinding of outcome assessments); (d) attrition bias (incomplete outcome data); (e) reporting bias (selective reporting); and (f) other bias (other sources of bias not addressed elsewhere). At the end of the assessment, each article will be categorized as 'high risk' or 'low risk.' When the risk of bias is reported as 'unclear' or as 'inadequate,' the author(s) of the article(s) will be contacted to clarify confusions or provide more information needed to take a stance on the status of 
bias in their work. Any disagreement between the two review authors concerning the risk of bias assessment will be discussed in a research meeting. Quality appraisal of the included studies will only be done after study selection has been completed, and during data extraction and synthesis. The strength of evidence for the findings of the review will be subsequently reported.

\section{RESULTS}

\section{Data synthesis and analysis, including assessment of heterogeneity}

The question of the effect of aerobic exercise intervention will be answered. In doing this, the review will examine, present, compare and pool in an evidence table study results on the effectiveness of intervention first on the primary outcome and followed by the secondary outcome. The effectiveness of aerobic exercise interventions will be established by conducting a meta-analysis of the effects (Pluye and Hong, 2014). Narrative synthesis will be employed when data from studies cannot be analyzed statistically.

\section{Statistical analysis}

The primary outcome will be analyzed before the secondary outcome. In case of a significant heterogeneity of study characteristics including diverse population studies, clinical characteristics of participants, diverse populations studied and intervention, study methodologies (study designs, statistical strategy, and outcome used), the heterogeneity will be assessed using the Cochran $X^{2}$ test, and further quantified using of $\mathrm{I}^{2}$ (Egger et al., 1997). This will guide our decision-making in the choice of effect model of meta-analysis to employ in this review. A meta-analysis using a random effect model will be used to pool together the data if the studies are homogenous in the study design and comparator (Higgins and Green, 2011). Narrative synthesis will be employed in analyzing and interpreting other heterogeneous studies following the recommendation of Centre for Reviews and Dissemination (Higgins and Green, 2011), specifically to explore the relationship in findings between and within the studies included.

In carrying out meta-analysis, the statistical approach will be to compare the absolute change in means to the baseline (and the 95\% CIs) proceeding intervention or with variation in the control groups where baseline data are available. Otherwise, the relative percentage changes between post intervention values in the intervention as well as control groups are compared. The risk of the outcome in the intervention group will be compared with the control group, with a risk difference calculated from the absolute difference between the treatment and control groups, or the relative risk (or equivalents) for outcome measures that are categorized as dichotomous. All adverse effects reported in the studies included will be reported. The continuous outcomes will be evaluated using the weighted mean difference or the standardized mean difference when different measurement scales are utilized to evaluate outcome. Review Manager (RevMan 5, Cochrane Collaboration, Oxford, UK) will be utilized for data analysis.

\section{Sensitivity analysis}

A subgroup analysis will be employed to study the potential influence on the treatment effect direction if many included studies had significant heterogeneity of study design, intervention type and setting. This will only be carried out if there are more than two studies, and not less than two of them are homogenous subset. Subgroup analysis will be limited to the primary outcome-serum lipid profile.

\section{Publication bias/meta-biases}

The funnel-plot for asymmetry and the Egger regression test (Egger et al., 1997) will be used to access the metabias. To achieve this, data from studies published only as abstracts will be included in the meta-analysis to see if they influence the direction of effect size.

\section{Confidence in cumulative evidence}

The quality of evidence of the included studies will be evaluated to determine the confidence in cumulative estimates in the systematic review. The GRADE (Grading of Recommendations Assessment, Development and Evaluation) will be utilized in judging the quality of evidence of the included studies (Guyatt et al., 2008). The quality of evidence will be assessed across the various domains: risk of bias, consistency, directness, precision and publication bias. Studies will be rated as (a) 'high quality' - where further research is unlikely to change the effect estimates, (b) 'moderate quality' -where further research is likely to have an important impact on the effect estimates or change the estimate, (c) 'low quality' - where further research is very likely to have an important impact on the effect estimates and change the estimate or, (d) 'very low quality' -where the estimate of effects is very uncertain.

\section{Reporting of the review}

The systematic review will be reported in synchrony with the PRISMA statement (Liberati et al., 2009). Reporting will be adapted to facilitate the inclusion of all items relevant to the review. A 
PRISMA checklist will be published alongside the final report.

\section{Potential amendments}

Given that quantitative studies are often influenced by publication bias, amendments will not be made on this review protocol because of the findings from the included studies. However, when it appears very justifiable to amend, such amendment(s) will be reported and implemented by the primary author. If amendment is necessary, a detailed report with reasons for amendment will be provided.

\section{Ethics}

The systematic review will involve data from already published studies, hence, ethical approval is not applicable.

This protocol was registered with the International Prospective Register of Systematic Reviews (PROSPERO, CRD42018091584).

\section{DISCUSSION}

Treatment involving the use of HAART in patients with HIV infection has been associated with increased risk of cardiovascular disease (CVD), due to altercation of metabolic processes leading to side effects such as lipodystrophy (abnormal fat displacement), hyperlipidemia (i.e., increased TGs and total cholesterol), decreased HDL-C among others (Jaggers et al., 2013). Extensive research has established the benefits of exercise as an appropriate intervention for reducing many modifiable risk factors of CVD, including a number of those that are elevated following HAART treatment (Jaggers et al., 2013). This systematic review will provide evidence to verify the hypothesis that aerobic exercise interventions can mitigate the adverse effects of HAART on serum lipid profile. The conclusion will stem from a synthesis of quantitative measurement of lipid profile parameters outcomes following aerobic exercise interventions. When done, the likelihood that the review will provide a coherent and reliable conclusion will depend on the quality and the quantity of evidence available in this subject matter. However, the review will elucidate the available evidence base on the effect of aerobic exercise intervention on HAART related dyslipidemia and provide impetus for use in a patient-centered care.

\section{CONFLICT OF INTEREST}

No potential conflict of interest relevant to this article was reported.

\section{REFERENCES}

Ahmed HM, Blaha MJ, Nasir K, Rivera JJ, Blumenthal RS. Effects of physical activity on cardiovascular disease. Am J Cardiol 2012;109:288-295.

Akers J, Aguiar-Ibáñez R, Baba-Akbari Sari A, Beynon S, Booth A, Burch J, Chambers D, Craig D, Dalton J, Duffy S, Eastwood A, Fayter D, Fonseca T, Fox D, Glanville J, Golder S, Hempel S, Light K, McDaid C, Norman G, Pierce C, Phillips B, Rice S, Rithalia A, Rodgers M, Sharp F, Sowden A, Stewart L, Stock C, Trowman R, Wade R, Westwood M, Wilson P, Woolacott N, Worthy G, Wright K. Systematic reviews: CRD's guidance for undertaking reviews in health care. York (UK): NHS Centre for Reviews and dissemination, University of York; 2009.

Anuurad E, Semrad A, Berglund L. Human immunodeficiency virus and highly active antiretroviral therapy-associated metabolic disorders and risk factors for cardiovascular disease. Metab Syndr Relat Disord 2009;7:401-410.

Carr A, Samaras K, Burton S, Law M, Freund J, Chisholm DJ, Cooper DA. A syndrome of peripheral lipodystrophy, hyperlipidaemia and insulin resistance in patients receiving HIV protease inhibitors. AIDS 1998; 12:F51-58

Cochrane Consumers and Communication Review Group. Communication review group: Data extraction template for Cochrane reviews [Internet]. London: Cochrane Collaboration; 2007 [updated 2016 Dec; cited 2017 May 10]. Available from: http://cccrg.cochrane.org/author-resources.

Egger M, Davey Smith G, Schneider M, Minder C. Bias in meta-analysis detected by a simple, graphical test. BMJ 1997;315:629-634.

Guyatt GH, Oxman AD, Vist GE, Kunz R, Falck-Ytter Y, Alonso-Coello P, Schünemann HJ; GRADE Working Group. GRADE: an emerging consensus on rating quality of evidence and strength of recommendations. BMJ 2008;336:924-926.

Higgins JP, Green S, editors. Cochrane handbook for systematic reviews of interventions. version 5.1.0 [Internet]. Oxford (UK): The Cochrane Collaboration, 2011 [uptated 2011 Mar; cited 2017 May 10]. Available from: www.cochrane-handbook.org.

Jaggers JR, Dudgeon W, Blair SN, Sui X, Burgess S, Wilcox S, Hand GA. A home-based exercise intervention to increase physical activity among people living with HIV: study design of a randomized clinical trial. BMC Public Health 2013;13:502.

Katzmarzyk PT, Leon AS, Wilmore JH, Skinner JS, Rao DC, Rankinen T, Bouchard C. Targeting the metabolic syndrome with exercise: evidence from the HERITAGE Family Study. Med Sci Sports Exerc 2003; 35:1703-1709.

Kelesidis T, Yang OO, Kendall MA, Hodis HN, Currier JS. Dysfunctional HDL and progression of atherosclerosis in HIV-1-infected and -unin- 
fected adults. Lipids Health Dis 2013;12:23.

Liberati A, Altman DG, Tetzlaff J, Mulrow C, Gøtzsche PC, Ioannidis JP, Clarke M, Devereaux PJ, Kleijnen J, Moher D. The PRISMA statement for reporting systematic reviews and meta-analyses of studies that evaluate health care interventions: explanation and elaboration. PLoS Med 2009;6:e1000100.

Moher D, Shamseer L, Clarke M, Ghersi D, Liberati A, Petticrew M, Shekelle P, Stewart LA; PRISMA-P Group. Preferred reporting items for systematic review and meta-analysis protocols (PRISMA-P) 2015 statement. Syst Rev 2015;4:1.

Palella FJ Jr, Delaney KM, Moorman AC, Loveless MO, Fuhrer J, Satten GA, Aschman DJ, Holmberg SD. Declining morbidity and mortality among patients with advanced human immunodeficiency virus infection. HIV Outpatient Study Investigators. N Engl J Med 1998;338:853860.

Pang MY, Eng JJ, Dawson AS, Gylfadóttir S. The use of aerobic exercise training in improving aerobic capacity in individuals with stroke: a meta-analysis. Clin Rehabil 2006;20:97-111.

Pluye P, Hong QN. Combining the power of stories and the power of numbers: mixed methods research and mixed studies reviews. Annu
Rev Public Health 2014;35:29-45.

Schambelan M, Benson CA, Carr A, Currier JS, Dubé MP, Gerber JG, Grinspoon SK, Grunfeld C, Kotler DP, Mulligan K, Powderly WG, Saag MS; International AIDS Society-USA. Management of metabolic complications associated with antiretroviral therapy for HIV-1 infection: recommendations of an International AIDS Society-USA panel. J Acquir Immune Defic Syndr 2002;31:257-275.

Shamseer L, Moher D, Clarke M, Ghersi D, Liberati A, Petticrew M, Shekelle P, Stewart LA; PRISMA-P Group. Preferred reporting items for systematic review and meta-analysis protocols (PRISMA-P) 2015: elaboration and explanation. BMJ 2015;350:g7647.

Souza SJ, Luzia LA, Santos SS, Rondó PH. Lipid profile of HIV-infected patients in relation to antiretroviral therapy: a review. Rev Assoc Med Bras (1992) 2013;59:186-198.

Thompson V, Medard B, Taseera K, Chakera AJ, Andia I, Emenyonu N, Hunt PW, Martin J, Scherzer R, Weiser SD, Bangsberg DR, Tien PC. Regional anthropometry changes in antiretroviral-naïve persons initiating a Zidovudine-containing regimen in Mbarara, Uganda. AIDS Res Hum Retroviruses 2011;27:785-791. 


\section{Appendix 1. Search terms}

\section{MEDLINE}

(((aerobic exercise OR exercises OR physical activit* OR acute exercise OR exercise training OR physical exertion OR Sport*)) AND (dyslipidemia OR dyslipoproteinemia OR hyperlipidemia OR lipids OR metabolic diseases OR lipid metabolism disorders OR hypercholesterolemia OR hyperlipoproteinemia OR hypertriglyceridemia OR lipoprot ?in)) AND (HIV OR seropositives OR haart OR antiretroviral therapy, highly active OR anti-hiv agents OR protease inhibito* OR non-nucleoside reverse transcriptase inhibitors) 